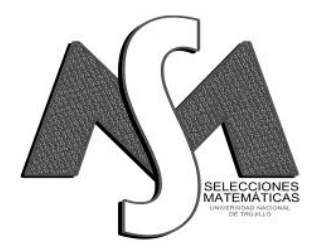

\title{
SELECCIONES MATEMÁTICAS
}

Universidad Nacional de Trujillo

http://revistas.unitru.edu.pe/index.php/SSMM

Vol. 03 (02): 49-59 (2016)

ISSN: 2411-1783 (versión electrónica)

\section{APROXIMACIÓN DE LA DISTANCIA EN LA ESFERA A TRAVÉS DE LA SOLUCIÓN NUMÉRICA DE UN PROBLEMA DE VALOR INICIAL ASOCIADO A GEODÉSICAS \\ APPROXIMATION OF THE DISTANCE IN THE SPHERE THROUGH OF THE NUMERICAL SOLUTION OF INITIAL VALUE PROBLEM ASSOCIATED TO GEODESICS}

\author{
FRANCO RUBIO * AND RONALD LEÓN **
}

Received: Aug 17, 2016

Accepted: Oct 08, 2016

\section{Resumen}

En este artículo, se plantea un algoritmo para aproximar la distancia Geodésica entre los puntos $p$ y $q$ de la esfera, mediante la solución numérica de un problema de valor inicial asociado al sistema de ecuaciones diferenciales ordinarias de las geodésicas; para lo cual se determina una dirección apropiada.

Palabras Clave: Distancia intrínseca, distancia geodésica, geodésicas, esfera, problema de valor inicial, aproximación.

\begin{abstract}
In this paper, we proposes an algorithm to approximate the Geodesic distance between points $p$ and $q$ of sphere, through the numerical solution of initial value problem associated with the system of ordinary differential equations of the geodesics; for this an appropriate direction is obtained.
\end{abstract}

Keywords: Intrinsec distance, geodesic distance, geodesics, sphere, initial value problem, approximation.

\footnotetext{
* Departamento de Matemáticas, Facultad de Ciencias Físicas y Matemáticas, Universidad Nacional de Trujillo, Av. Juan Pablo II s/n, Ciudad Universitaria, Trujillo-Perú (frubio@unitru.edu.pe)

** Departamento de Matemáticas, Facultad de Ciencias Físicas y Matemáticas, Universidad Nacional de Trujillo, Av. Juan Pablo II s/n, Ciudad Universitaria, Trujillo-Perú (rleon@unitru.edu.pe)
} 


\section{Introduction}

The problem of finding the distance between two points on a regular surface $S$ (connected and complete), in general it is not easy; since it requires to find the minimal geodesic that joins these points. An algorithm called Leap-Frog (Kanya, Noakes, 1997-1998) was developed to approximate geodesics; and Noakes in 1998 developed a global algorithm for this purpose.

In the case of a sphere, without loss of generality, we can consider the unit sphere centered on the origin of coordinates, the distance between two points, is found using the maximum circumference that passes through these points; since in a sphere the maximum circumferences are the geodesics. This distance in the sphere is known as the geodesic distance or the shortest arc distance between points (Wesolowsky, 1982).

In this paper, we obtain a numerical approximation to the geodesic distance, solving a problem of initial value associated to the system of ordinary differential equations of the geodesics; based on the method developed by Rubio (2015). For this, we obtain a vector that will give the direction that will solve the problem of initial value respective. An algorithm is also provided for this purpose.

\section{Regular Surfaces.}

In this section we enunciate some results on Differential Geometry, which were taken of the book of Do Carmo (1976).

Definition 2.1. A subset $S \subset R^{3}$ is a Regular Surface if, for each $p \in S$, there exists a neighborhood $V$ in $R^{3}$ and a map $X: U \subset R^{2} \rightarrow V \cap S$ of an open set $U \subset R^{2}$ onto $V \cap S \subset R^{3}$ such that:

1. $X \in C^{\infty}(U)$.

2. $X$ is a homeomorphism.

3. For each $q \in U$, the differential $d X_{q}: R^{2} \rightarrow R^{3}$ is one-to-one.

The mapping $X$ is called a parametrization of $\mathrm{S}$; and in coordinates it is given for

$$
X(u, v)=(x(u, v), y(u, v), z(u, v)), \forall(u, v) \in U .
$$

Definition 2.2. A nonconstant, parametrized curve $\alpha: I \subset R \rightarrow S$ is called parametrized Geodesic if:

$$
\frac{D}{d t}\left(\frac{d \alpha}{d t}(t)\right)=0, \forall t \in I
$$

where $\frac{D}{d t}$ denotes the Covariant Derivative.

Now, let's consider a parametrization $X: U \subset R^{2} \rightarrow S$, which that $X(U) \cap \alpha(I) \neq \emptyset$. Also, the parametrization induce a base $\left\{X_{u}(q), X_{v}(q)\right\}$ in the tangent space $T_{p} S$, to $S$ at $p=X(q)$. 
Now, let $W(t)$ be a vectorial field tangent along a curve differentiable parametrized $a: I \subset R \rightarrow S$. The expressions of field $W(t)$ in the parametrization is:

$$
W(t)=a(t) X_{u}(u(t), v(t))+b(t) X_{v}(u(t), v(t))
$$

The expressions of covariant derivative of field $W(t)$, by (2), is:

$$
\begin{aligned}
\frac{D}{d t} W(t)= & \left(a^{\prime}+\Gamma_{11}^{1} a u^{\prime}+\Gamma_{12}^{1} a v^{\prime}+\Gamma_{12}^{1} b u^{\prime}+\Gamma_{22}^{1} b v^{\prime}\right) X_{u}+ \\
& +\left(b^{\prime}+\Gamma_{11}^{2} a u^{\prime}+\Gamma_{12}^{2} a v^{\prime}+\Gamma_{12}^{2} b u^{\prime}+\Gamma_{22}^{2} b v^{\prime}\right) X_{v}
\end{aligned}
$$

where the $\Gamma_{i j}^{k}, \forall i, j, k=1,2$, are called the Christoffel symbols which are given by:

$$
\begin{array}{cc}
\Gamma_{11}^{1}=\frac{G E_{u}-2 F F_{u}+F E_{v}}{2\left(E G-F^{2}\right)} \quad, & \Gamma_{11}^{2}=\frac{2 E F_{u}-E E_{v}-F E_{u}}{2\left(E G-F^{2}\right)} \\
\Gamma_{12}^{1}=\frac{G E_{v}-F G_{u}}{2\left(E G-F^{2}\right)} & , \quad \Gamma_{12}^{2}=\frac{E G_{u}-F E_{v}}{2\left(E G-F^{2}\right)} \\
\Gamma_{22}^{1}=\frac{2 G F_{v}-G G_{u}-F G_{v}}{2\left(E G-F^{2}\right)} \quad, \quad \Gamma_{22}^{2}=\frac{E G_{v}-2 F F_{v}+F G_{u}}{2\left(E G-F^{2}\right)}
\end{array}
$$

Also, the coefficients of the First Fundamental Form of $\mathrm{S}$ in the parameterization $\mathrm{X}$, are given by:

$E(u, v)=\left\langle X_{u}(u, v), X_{u}(u, v)\right\rangle, \quad F(u, v)=\left\langle X_{u}(u, v), X_{v}(u, v)\right\rangle, \quad G(u, v)=\left\langle X_{v}(u, v), X_{v}(u, v)\right\rangle(5)$

If $\alpha: I \subset R \rightarrow S$ is parametrized geodesic, its expressions in the parametrization is given by:

$$
\alpha(t)=X(u(t), v(t))
$$

Therefore, the tangent vector is given by:

$$
\frac{d \alpha}{d t}(t)=X_{u} u^{\prime}(t)+X_{v} v^{\prime}(t)
$$

Using (1) and (3) for $W(t)=\frac{d \alpha}{d t}(t)$; its have:

$$
\left\{\begin{array}{l}
u^{\prime \prime}+\Gamma_{11}^{1}\left(u^{\prime}\right)^{2}+2 u^{\prime} v^{\prime} \Gamma_{12}^{1}+\Gamma_{22}^{1}\left(v^{\prime}\right)^{2}=0 \\
v^{\prime \prime}+\Gamma_{11}^{2}\left(u^{\prime}\right)^{2}+2 u^{\prime} v^{\prime} \Gamma_{12}^{2}+\Gamma_{22}^{2}\left(v^{\prime}\right)^{2}=0
\end{array}\right.
$$

that is a system of ordinary differential equations of second order. 


\section{Geodesic on $S^{2}$}

Consider the unit sphere:

$$
S^{2}=\left\{(x, y, z) \in \mathbb{R}^{3} / x^{2}+y^{2}+z^{2}=1\right\}
$$

The upper hemisphere or northern hemisphere is given by:

$$
S_{+}^{2}=\left\{(x, y, z) \in \mathbb{R}^{3} / z=\sqrt{1-x^{2}-y^{2}}, x^{2}+y^{2}<1\right\}
$$

which is the graph of the differentiable function $f: U \subset \mathbb{R}^{2} \rightarrow \mathbb{R}$ defined by

$$
f(x, y)=\sqrt{1-x^{2}-y^{2}} \text {, donde } U=\left\{(x, y) \in \mathbb{R}^{2}: x^{2}+y^{2}<1\right\} .
$$

A parametrization for (8) is given by $X: U \subset \mathbb{R}^{2} \rightarrow S_{+}^{2}$ defined by:

$$
X(u, v)=\left(u, v, \sqrt{1-u^{2}-v^{2}}\right)
$$

The coefficients of the first fundamental form (5) are:

$$
E=1+\frac{u^{2}}{1-u^{2}-v^{2}}, \quad F=\frac{u v}{1-u^{2}-v^{2}}, \quad G=1+\frac{v^{2}}{1-u^{2}-v^{2}}
$$

Its derivatives:

$$
\left\{\begin{aligned}
E_{u} & =\frac{2 u-2 u v^{2}}{\left(1-u^{2}-v^{2}\right)^{2}}, & E_{v} & =\frac{2 u^{2} v}{\left(1-u^{2}-v^{2}\right)^{2}} \\
F_{u} & =\frac{v-v^{3}+u^{2} v}{\left(1-u^{2}-v^{2}\right)^{2}}, & F_{v} & =\frac{u-u^{3}+u v^{2}}{\left(1-u^{2}-v^{2}\right)^{2}} \\
G_{u} & =\frac{2 u v^{2}}{\left(1-u^{2}-v^{2}\right)^{2}}, & G_{v} & =\frac{2 v-2 u^{2} v}{\left(1-u^{2}-v^{2}\right)^{2}}
\end{aligned}\right.
$$

Using (10) and (11), the Christoffel symbols $\Gamma_{i j}^{k}, \forall i, j, k=1,2$. are obtained.

Therefore, the initial value problem associated with the geodesics is:

$$
\left\{\begin{array}{c}
u^{\prime \prime}+\Gamma_{11}^{1}\left(u^{\prime}\right)^{2}+2 u^{\prime} v^{\prime} \Gamma_{12}^{1} a v^{\prime}+\Gamma_{22}^{1}\left(v^{\prime}\right)^{2}=0 \\
v^{\prime \prime}+\Gamma_{11}^{2}\left(u^{\prime}\right)^{2}+2 u^{\prime} v^{\prime} \Gamma_{12}^{2} a v^{\prime}+\Gamma_{22}^{2}\left(v^{\prime}\right)^{2}=0 \\
u(0)=u_{0}, \quad v(0)=v_{0} \\
u^{\prime}(0)=\tau_{1}, \quad v^{\prime}(0)=\tau_{2}
\end{array}\right.
$$

where $\tau=\left(\tau_{1}, \tau_{2}\right) \in R^{2}$. 


\section{Distance on $S^{2}$}

Since the Gaussian Curvature of $S^{2}$ is $K=1 \neq 0$, the formula for find the Euclidean distance in the plane does not apply in this case; for that reason, the distance between two points in $S^{2}$, is calculated through the Intrinsic distance $d_{S}$ of the connected regular surface $S$; which is defined by:

$$
d_{S}(p, q)=\inf \left\{l(\alpha)=\int_{0}^{1}\left\|\alpha^{\prime}(t)\right\| d t / \alpha \in C_{p q}\right\}
$$

where:

$$
C_{p q}=\{\alpha:[0,1] \rightarrow S / \alpha(0)=p, \alpha(1)=q\}
$$

In the case of $S^{2}$, the geodesics are maximum circumferences; therefore, the shortest distance between any two points of $S^{2}$ is measured along a maximum circle passing through them.

According to (Wesolowsky, 1982), this distance is known as shorter arc distance.

Mangalica (2005), and Wesolowski (1982), consider the spherical coordinates $(\varnothing, \theta)$, a point $p \in S^{2}$ is defined by its latitude $\emptyset$ and longitude $\theta$, and is denoted by $p=X(\varnothing, \theta)$, where

$$
-\frac{\pi}{2}<\varnothing<\frac{\pi}{2}, \quad 0<\theta<2 \pi .
$$

Thus, when considering two points $p_{1}=X\left(\emptyset_{1}, \theta_{1}\right), p_{2}=X\left(\emptyset_{2}, \theta_{2}\right)$ on $S^{2}$, the shortest length arc $\alpha=\operatorname{arc}\left(p_{1}, p_{2}\right)$, satisfies:

$$
\begin{gathered}
l(\alpha)=\cos ^{-1}\left[\cos \emptyset_{1} \cos \emptyset_{2} \cos \left(\theta_{1}-\theta_{2}\right)+\sin \emptyset_{1} \sin \emptyset_{2}\right] \\
l(\alpha)=\cos ^{-1}\left[<p_{1}, \quad p_{2}>\right]
\end{gathered}
$$

which is called (Donnay, 1945) geodesic distance, denoted by $d_{g}\left(p_{1}, p_{2}\right)$, between the points $p_{1}$ and $p_{2}$.

\section{The Geodesic Direction}

In this paper we will approximate the distance $d_{S}$ or geodesic distance, solving a problem of initial value associated to the system of ordinary differential equations of geodesics (12).

Definition 5.1. The vector $\tau=\left(\tau_{1}, \tau_{2}\right) \in \mathbb{R}^{2}$ given by (12), which allows to determine the geodesic distance, is called Minimal Geodesic Direction.

Theorem 5.2. Let $p_{0}, p_{1} \in S_{+}^{2}, p_{0} \neq p_{1}$. A tangent vector $T$ to $S_{+}^{2}$ in $p_{0}$, in the direction of $p_{1}$, is given by:
a) $=p_{0} x\left(p_{0} x p_{1}\right)$, or
b) $T=-p_{0} x\left(p_{0} \times p_{1}\right)$ 


\section{Proof}

As $p_{0}, p_{1} \in S_{+}^{2}$, we denote with $p_{0}$ and $p_{1}$ the associated vector radius to $p_{0}$ and $p_{1}$, respectively.

Furthermore, as $p_{0} \neq p_{1}$, then $p_{0}$ and $p_{1}$ are linearly independent; and as in $S^{2}$, the geodesics are maximum circumferences, then they are in the plane generated by $p_{0} y p_{1}$.

Denoting by:

$\wp_{01}$ the plane generated by $p_{0}$ and $p_{1}$.

$\mathbb{C}_{01}$ maximum semicircle passing through $p_{0}$ and $p_{1}$.

Thus $\mathbb{C}_{01} \subset \wp_{01}$. Then, the vector cross produc of $p_{0}$ and $p_{1}$ :

$$
p_{0} \times p_{1} \perp \wp_{01}
$$

Thus, a vector tangent to $S_{+}^{2}$, which starts from $p_{0}$ and is the plane $\wp_{01}$, is:

$$
p_{0} \times\left(p_{0} \times p_{1}\right)
$$

Let $w=p_{1}-p_{0}$, then:

a) If $\left\langle w, p_{0} x\left(p_{0} x p_{1}\right)\right\rangle>0$, then $T=p_{0} x\left(p_{0} x p_{1}\right)$.

b) If $\left\langle w, p_{0} x\left(p_{0} x p_{1}\right)\right\rangle<0$, then $T=-p_{0} x\left(p_{0} x p_{1}\right)$.

Theorem 5.3. Let $p_{0}, p_{1} \in S_{+}^{2}, p_{0} \neq p_{1}$. The tangent vector $T=\left(T_{x}, T_{y}, T_{z}\right)$ to $S_{+}^{2}$ in $p_{0}$, given by (16) or (17) in coordinates is respectively:

$$
\left\{\begin{array}{c}
T_{x}= \pm\left(v_{0} P_{z}-P_{y} \sqrt{1-u_{0}^{2}-v_{0}^{2}}\right) \\
T_{y}= \pm\left(P_{x} \sqrt{1-u_{0}^{2}-v_{0}^{2}}-u_{0} P_{z}\right) \\
T_{z}= \pm\left(u_{0} P_{y}-v_{0} P_{x}\right)
\end{array}\right.
$$

where $p_{0} x p_{1}=\left(P_{x}, P_{y}, P_{z}\right)$.

\section{Proof}

We only give the proof respect to (16); because the other option is analogous.

Using the parametrization (9), there are $q_{0}=\left(u_{0}, v_{0}\right), q_{1}=\left(u_{1}, v_{1}\right)$ in $U$, such that:

$$
\left\{\begin{array}{c}
p_{0}=X\left(u_{0}, v_{0}\right), p_{1}=X\left(u_{1}, v_{1}\right) \\
p_{0}=\left(u_{0}, v_{0}, \sqrt{1-u_{0}^{2}-v_{0}^{2}}\right), \\
p_{1}=\left(u_{1}, v_{1}, \sqrt{1-u_{1}^{2}-v_{1}^{2}}\right)
\end{array}\right.
$$


Using (20), we have: $p_{0} x p_{1}=\left(P_{x}, P_{y}, P_{z}\right)$, where:

$$
\left\{\begin{array}{c}
P_{x}=v_{0} \sqrt{1-u_{1}^{2}-v_{1}^{2}}-v_{1} \sqrt{1-u_{0}^{2}-v_{0}^{2}} \\
P_{y}=u_{1} \sqrt{1-u_{0}^{2}-v_{0}^{2}}-u_{0} \sqrt{1-u_{1}^{2}-v_{1}^{2}} \\
P_{z}=u_{0} v_{1}-u_{1} v_{0}
\end{array}\right.
$$

Therefore, from (21), the vector $T=\left(T_{x}, T_{y}, T_{z}\right)=p_{0} x\left(p_{0} x p_{1}\right)$ is given by:

$$
\left\{\begin{array}{c}
T_{x}=v_{0} P_{z}-P_{y} \sqrt{1-u_{0}^{2}-v_{0}^{2}} \\
T_{y}=P_{x} \sqrt{1-u_{0}^{2}-v_{0}^{2}}-u_{0} P_{z} \\
T_{z}=u_{0} P_{y}-v_{0} P_{x}
\end{array}\right.
$$

The following theorem allows us to find the minimal geodesic direction; which is used in the initial condition of the initial value problem (12).

Theorem 5.4. The minimal geodesic direction $\tau=\left(\tau_{1}, \tau_{2}\right) \in \mathbb{R}^{2}$, for the system (12), is given by:

$$
\left\{\begin{array}{l}
\tau_{1}=v_{0} P_{z}-P_{y} \sqrt{1-u_{0}^{2}-v_{0}^{2}} \\
\tau_{2}=P_{x} \sqrt{1-u_{0}^{2}-v_{0}^{2}}-u_{0} P_{z}
\end{array}\right.
$$

\section{Proof}

As $S_{+}^{2}$ is the graph of the differentiable function $f(x, y)=\sqrt{1-x^{2}-y^{2}}$, of theorem (5.3), we have:

$$
\left\{\begin{array}{c}
T_{x}=v_{0} P_{z}-P_{y} \sqrt{1-u_{0}^{2}-v_{0}^{2}} \\
T_{y}=P_{x} \sqrt{1-u_{0}^{2}-v_{0}^{2}}-u_{0} P_{z} \\
T_{z}=u_{0} P_{y}-v_{0} P_{x}
\end{array}\right.
$$

Therefore, the vector $\tau=\left(\tau_{1}, \tau_{2}\right) \in \mathbb{R}^{2}$, is given by:

$$
\left\{\begin{array}{l}
\tau_{1}=v_{0} P_{z}-P_{y} \sqrt{1-u_{0}^{2}-v_{0}^{2}} \\
\tau_{2}=P_{x} \sqrt{1-u_{0}^{2}-v_{0}^{2}}-u_{0} P_{z}
\end{array}\right.
$$

Because it is the projection of $T=\left(T_{x}, T_{y}, T_{z}\right)$ on the coordinate plane XOY. 


\section{Algorithm.}

In this section we developed a algorithm (Rubio, 2015), to approximate the Geodesic distance $d_{g}\left(p_{0}, p_{1}\right), p_{0}, p_{1} \in S_{+}^{2}$, Obtaining the numerical solution of I.V.P (12).

1. Input $p_{0}=\left(u_{0}, v_{0}, \sqrt{1-u_{0}^{2}-v_{0}^{2}}\right), p_{1}=\left(u_{1}, v_{1}, \sqrt{1-u_{1}^{2}-v_{1}^{2}}\right), N$, tol.

2. $h=\frac{1}{N}, \quad t_{i}=i h, \quad i=0,1, \ldots, N$,

3. Using (22), calculate:

$$
\tau=\left(\tau_{1}, \tau_{2}\right) \in \mathbb{R}^{2}
$$

4. $u(0)=u_{0}, v(0)=v_{0}$,

$u^{\prime}(0)=\tau_{1}, \quad v^{\prime}(0)=\tau_{1}$

5.Solver the I.V.P (12),

$$
\text { Error }=\sqrt{\left(u(k)-u_{1}\right)^{2}+\left(v(k)-v_{1}\right)^{2}}
$$

5.1. If Error $\leq$ tol, then

Calculate $d_{a p}$, where $d_{a p}$ is the distance obtained by the algorithm.

6. $d_{g}\left(p_{0}, p_{1}\right) \approx d_{a p}$.

7. End

\section{Examples.}

7.1. Find the geodesic distance between points $p_{0}, p_{1} \in S^{2}$, given by:

$$
p_{0}=(0.7,0.2,0.6856) \text { y } p_{1}=(0.12,0.83,0.5447) .
$$

Let:

$d_{a p}$ approximate distance given by the algorithm.

$d_{g}$ geodesic distance obtained by (15).

Using the algorithm we obtain:

\begin{tabular}{|c|c|c|c|c|c|c|c|c|}
\hline \multicolumn{3}{|c|}{$p_{0}$} & \multicolumn{3}{c|}{$p_{1}$} & Distance: $d_{a p}$ & $\begin{array}{l}\text { Geodesic } \\
\text { distance: } d_{g}\end{array}$ & Error: $\left|d_{a p}-d_{g}\right|$ \\
\hline 0.7 & 0.2 & 0.6856 & 0.12 & 0.83 & 0.5447 & 0.8913 & 0.8977 & 0.0064 \\
\hline
\end{tabular}

Table Nro. 1. Comparison between distance $d_{a p}$ and distance $d_{g}$. 


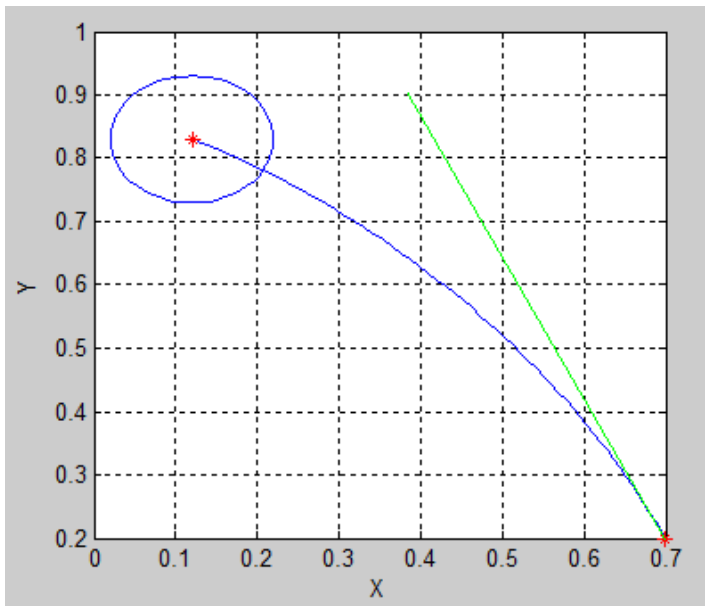

Fig. Nro. 1. Pre-image of the geodesic in the plane, and circle of arrival.

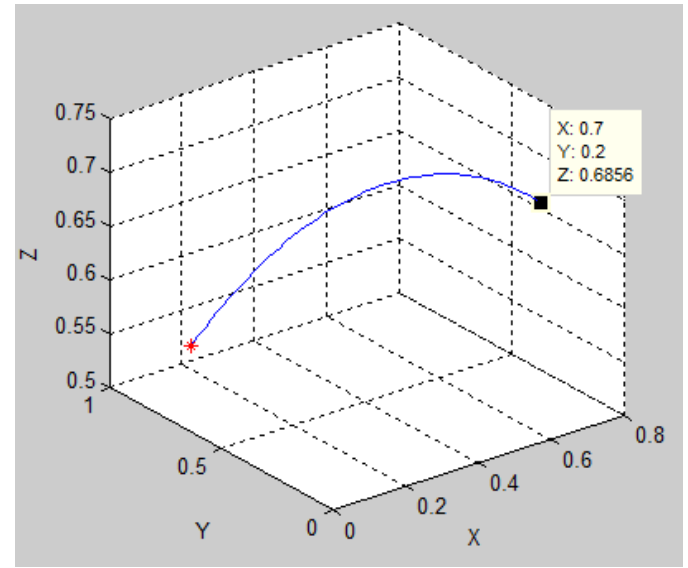

Fig. Nro. 2. Geodesic from $P_{0}$ to $P_{1}$.

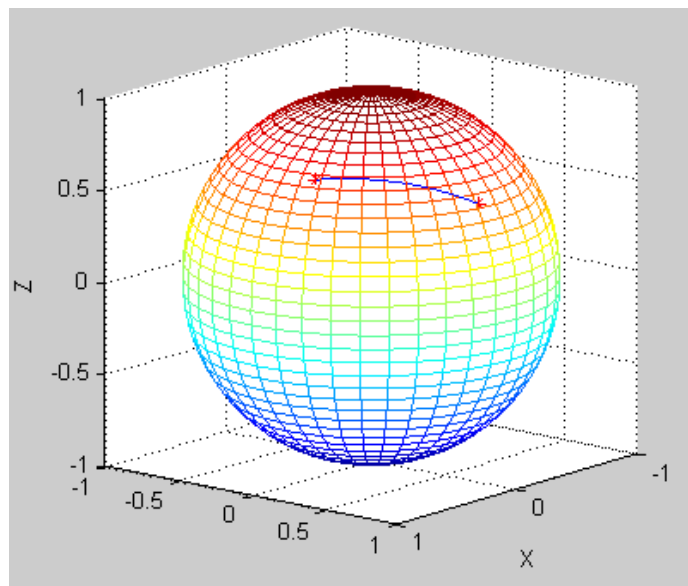

Fig. Nro. 3. Geodesic from $P_{0}$ to $P_{1}$. 
7.2 The following table shows results.

\begin{tabular}{|c|c|c|c|c|c|c|c|c|}
\hline \multicolumn{6}{|c|}{ Points on $S_{+}^{2}$} & \multicolumn{3}{|c|}{ Parameter: $\mathrm{N}=200$, tol $=0.01, \mathrm{~h}=4 / \mathrm{N}$} \\
\hline \multicolumn{3}{|c|}{$p_{0}$} & \multicolumn{3}{|c|}{$p_{1}$} & $\begin{array}{c}\text { Distance: } \\
d_{a p}\end{array}$ & $\begin{array}{c}\text { Geodesic } \\
\text { Distance: } \\
d_{g}\end{array}$ & $\begin{array}{l}\text { Error: } \\
\mid d_{a p}-d_{q}\end{array}$ \\
\hline 0.7 & 0.2 & 0.6856 & 0.12 & 0.83 & 0.5447 & 0.8913 & 0.8977 & 0.0064 \\
\hline 0.13 & 0.17 & 0.9768 & 0.77 & -0.32 & 0.5520 & 0.9409 & 0.9460 & 0.0051 \\
\hline 0.45 & -0.54 & 0.7113 & -0.69 & 0.55 & 0.4705 & 1.8279 & 1.8471 & 0.0192 \\
\hline-0.3 & -0.6 & 0.7416 & -0.21 & -0.08 & 0.9744 & 0.5854 & 0.5851 & 0.0003 \\
\hline 0.5 & -0.5 & 0.7071 & -0.7 & -0.7 & 0.1414 & 1.4726 & 1.4706 & 0.002 \\
\hline-0.62 & 0.43 & 0.6563 & 0.39 & -0.47 & 0.7918 & 1.4957 & 1.4950 & 0.0007 \\
\hline 0.0 & -0.8 & 0.6000 & 0.0 & 0.9 & 0.4359 & 2.0262 & 2.0471 & 0.0209 \\
\hline 0.47 & 0.66 & 0.5861 & 0.21 & -0.72 & 0.6614 & 1.5599 & 1.5596 & 0.0003 \\
\hline 0.9 & -0.1 & 0.4243 & $\begin{array}{l}-0.8 \\
\end{array}$ & -0.58 & 0.1536 & 2.1985 & 2.2103 & 0.0118 \\
\hline-0.5 & 0.8 & 0.3317 & 0.8 & 0.21 & 0.5620 & 1.6183 & 1.6164 & 0.0019 \\
\hline 0.49 & 0.0 & 0.4359 & -0.9 & 0.0 & 0.4359 & 2.2282 & 2.2395 & 0.0113 \\
\hline 0.2 & 0.15 & 0.9682 & 0.8 & -0.23 & 0.5542 & 0.8394 & 0.8472 & 0.0078 \\
\hline-0.2 & -0.15 & 0.9682 & -0.8 & 0.17 & 0.5754 & 0.7945 & 0.8070 & 0.0125 \\
\hline 0.0 & 0.0 & 1.000 & 0.0 & 0.98 & 0.1990 & 1.3328 & 1.3705 & 0.0377 \\
\hline 0.91 & -0.1 & 0.4024 & -0.1 & -0.9 & 0.4243 & 1.3994 & 1.4003 & 0.0009 \\
\hline-0.2 & 0.85 & 0.4873 & 0.33 & 0.37 & 0.8684 & 0.8297 & 0.8343 & 0.0046 \\
\hline
\end{tabular}

Table Nro. 2. Comparison between distance $d_{a p}$ and distance $d_{g}$.

\section{References}

[1] Kaya, C. Y, and Noakes, J. L. The Leap-Frog Algorithm and Optimal Control: theoretical aspect.Proceedings of ICOTA 98, Perth, Australia.

[2] Kaya, C. Y, and Noakes, J. L. Geodesic and an Optimal Control Algorithm, Proccedings of the 36 th IEEE CD6, San Diego, California, U.S.A, December 1997.

[3] Noakes, J. L. A Global Algorithm for Geodesics, Journal of the Australian Mathematical Society, 1998.

[4] Wesolowsky, G. O. Location problems on a sphere. Regional Science and Urban Economics, 1982, vol. 12, no 4, p. 495-508. 
[5] Rubio, Franco. Aproximación de la distancia en el plano a través de la solución numérica de problemas de valor inicial asociados a Geodésicas. Selecciones Matemáticas, 2015, vol. 2, no 02, p. 81-91.

[6] Do Carmo, Manfredo. Differential geometry of curves and surfaces. Englewood Cliffs: Prentice-hall, 1976.

[7] Mangalika, Dedigama. Spherical Location Problems with Restricted Regions and Polygonal Barriers. 2005.

[8] Donnay, Joseph D. Spherical trigonometry. Read Books Ltd, 2013. 